$\alpha$-amylase isoenzymes

cystic fibrosis pancreas

salivary secretion

\title{
Serum Amylase Isoenzymes in Cystic Fibrosis Patients. Evidence for a Generalized Defect in Exocrine Gland Secretory Regulation
}

\author{
BAIBA KURINS GILLARD, ${ }^{(27)}$ JANET A. SIMBALA, AND STEPHEN A. FEIG \\ Gwynne Hazen Cherry Memorial Laboratories, Department of Pediatrics, UCLA School of Medicine, Los Angeles, \\ California, USA
}

\begin{abstract}
Summary
Altered exocrine gland function is characteristic of cystic fibrosis (CF). This study was undertaken to determine the relationship between pancreatic and salivary exocrine dysfunction in $\mathrm{CF}$. We have measured serum pancreatic $(P)$ and salivary-like $(S)$ isoenzyme concentrations in $\mathrm{CF}$ patients, their obligate heterozygote parents and normal controls $(\mathbf{N})$ using a quantitative gel-electrophoretic method. Our results show that serum levels of these isoenzymes correlate with the respective pancreatic and salivary gland amylase output and, therefore, can be used as measures of pancreatic and salivary exocrine function. Thus, $P$ isoenzyme levels are low in CF patients with pancreatic insufficiency (mean \pm S.D.: $1.3+0.6$ starch units per liter versus $5.8 \pm 1.7$ for the $N$ group, $P<0.001$ ). In contrast, $P$ isoenzyme levels are elevated in $C F$ patients with adequate pancreatic function $(12.0 \pm 5.3 ; P<$ 0.002 versus $N)$. Whereas $P$ isoenzyme levels differentiate $C F$ patients with pancreatic insufficiency from those with adequate pancreatic function, $\mathbf{S}$ isoenzyme levels are equally elevated irrespective of pancreatic function $(12.5 \pm 5.3$ and $12.5 \pm 5.2$ versus $8.1 \pm 2.7$ for the $N$ group, $P<0.001$ and $<0.01$, respectively). Thus, increased salivary amylase levels in $\mathrm{CF}$ patients are not due to a compensatory mechanism for reduced pancreatic amylase output, but rather are intrinsic to CF. In those patients with adequate pancreatic function, the increased $S$ isoenzyme levels correlate with increased $P$ levels $(r=0.72)$, indicating defective control of both pancreatic and salivary exocrine secretion in $C F$. In obligate heterozygote samples, $P$ isoenzyme levels are increased $(7.6 \pm 2.5 ; P<0.05$ versus $N)$ whereas $S$ isoenzyme levels are normal $(8.2 \pm 5.6)$, indicating that this defect is partially expressed in the carrier state. We conclude that elevated saliva amylase and altered serum amylase isoenzyme levels in the $C F$ patient and carrier state are due to a generalized defect in exocrine gland secretory regulation in $\mathrm{CF}$.
\end{abstract}

\section{Speculation}

We speculate that the altered exocrine secretion in cystic fibrosis patients and their parents is due to the presence of an extra, cystic-fibrosis-associated, stimulatory agent and that the exocrine pancreas is more sensitive to this agent than are the salivary glands.

Cystic fibrosis (CF) is the most common lethal genetic disease of Caucasians. Although the basic pathophysiologic defect has not been determined, the clinical triad of chronic pulmonary disease, pancreatic insufficiency, and increased sweat electrolyte concentration has been ascribed to a defect in the control of exocrine gland secretion (6).

Our previous work $(8,9)$ and that of others $(2,6)$ has shown that amylase activity ( $\alpha$-D-glucan 4 -glucanohydrolase, EC 3.2.1.1) is increased in saliva of $\mathrm{CF}$ patients. This increased activity could be due to a primary defect in regulation of exocrine secretion in $\mathrm{CF}$ or be a compensatory mechanism for the decreased pancreatic amylase secretion in the $80 \%$ of CF patients with pancreatic insufficiency. Altered exocrine function of the pancreatic or salivary glands is reflected in alterations of the respective pancreatic (P) or salivary-like (S) amylase isoenzyme concentrations in serum $(1,14,22)$. Therefore, to differentiate between the two hypotheses, we have measured serum amylase isoenzyme levels and determined the relationship between salivary and pancreatic amylase output in the CF genotype.

\section{MATERIALS AND METHODS}

Specimens were collected from patients with CF (age, 5 to 35 years), their obligate heterozygote parents $(\mathrm{H})$ (age, 26 to 47 years) and normal control donors (N) (age, 5 to 47 years) after obtaining informed consent. Blood was obtained by venipuncture and was transferred to red-top vacutainers, allowed to clot, and centrifuged to obtain the serum. Whole saliva $(3$ to $5 \mathrm{ml}$ ) was collected without a stimulant into plastic tubes and centrifuged at $5000 \times g$ for 15 min to obtain a clear supernate. Samples were stored at $-20^{\circ} \mathrm{C}$ until assayed.

CF patients were divided into two groups on the basis of clinical and laboratory assessment of pancreatic function. Group $A$ had pancreatic insufficiency, and group $B$ had adequate pancreatic function. The clinical diagnosis of pancreatic insufficiency in $C F$ patients was based on a history of steatorrhea, low serum carotene levels, and the need for enzyme replacement therapy. Pancreatic insufficiency was further confirmed in six patients by pancreatic secretory studies with duodenal intubation as described by Cox $e t$ al. (3). Adequate pancreatic function was defined by absence of clinical evidence of pancreatic insufficiency and either no use $(6$ patients) or minimal use ( 2 patients) of enzyme replacement therapy.

Total serum and saliva amylase activities were determined by a saccharogenic assay as previously described $(7,8)$. Activity is expressed in starch units/liter. One starch unit is equal to 5.7 Somogyi units (13) and 10.6 IU (16).

Serum amylase can be separated by polyacrylamide gel electrophoresis into two principal groups: $P$ isoenzymes and $S$ isoenzymes (7). The $P$ isoenzymes are derived from the pancreas (1). The primary source of the $S$ isoenzymes are the salivary glands, but other organs may also contribute to this fraction $(1,16)$.

The $S$ and $P$ isoenzymes in serum were separated and quantitated as recently described (7). Briefly, after electrophoresis of serum on $7.2 \%$ polyacrylamide gel, the gel was incubated in phosphate-buffered starch solution and stained with $I_{2} / K I$. The relative activities of the $S$ and $P$ isoenzymes were quantitated by densitometry. This method can measure activity as low as 0.2 starch unit/liter/band. It has a reproducibility $\mathrm{CV}=0.04$ and a precision $\mathrm{CV}=0.03$ for normal samples (7). 


\section{RESULTS}

The separation of serum amylase isoenzymes on polyacrylamide gels is illustrated in Figure 1 . Normal serum contains $\mathbf{S}$ and $\mathrm{P}$ bands of about equal intensity (Fig. $1 A$ ). CF patients with pancreatic insufficiency have a normal $S$ band, but a very faint $P$ band (Fig. $(B)$. A variant pattern, with a second band of pancreatic origin trailing the usual $\mathrm{P}$ band (Fig. IC) (12) was also observed.

To establish that the serum levels of $P$ isoenzyme were related to pancreatic amylase output, $P$ levels in patients who had pancreatic insufficiency confirmed by duodenal intubation were compared to levels in patients who did not use any enzyme replacement therapy. The data is summarized in Table 1. All six study patients with confirmed pancreatic insufficiency (group A) had very low $P$ isoenzyme levels. The six study patients who had clinically normal pancreatic function (group B) had much higher serum $\mathrm{P}$ isoenzyme activity. These results, confirming earlier studies $(22,24)$ indicate that serum $P$ isoenzyme levels are a good measure of pancreatic exocrine function in CF.

To establish that serum $\mathbf{S}$ isoenzyme levels are related to saliva amylase levels, serum $\mathbf{S}$ isoenzyme and saliva amylase were determined in 51 pairs of serum and saliva samples from $C F, H$, and $\mathrm{N}$ donors. A scatter plot of the data is shown in Figure 2. Linear regression analysis gives a correlation coefficient of $r=0.50 ; P<$ 0.001 . Thus, there is a highly significant correlation between serum $\mathrm{S}$ isoenzyme activity and saliva amylase activity. We conclude

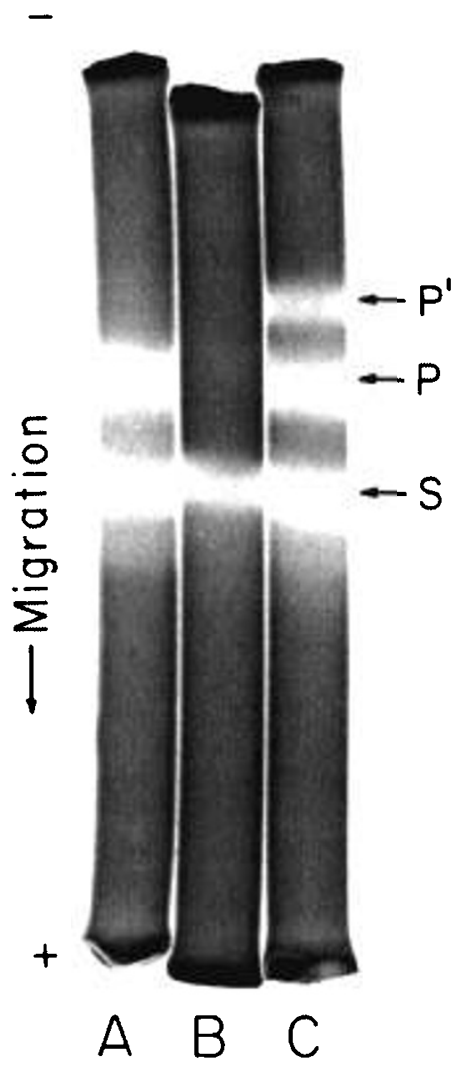

Fig. I. Isoenzyme banding patterns. Serum amylase isoenzyme banding patterns on $7.2 \%$ polyacrylamide gels are illustrated. Pancreatic $\left(P, P^{\prime}\right)$ and $S$ bands are indicated. Gel $A$, normal serum; gel $B$, serum from patient with $C F$ and pancreatic insufficiency; gel $C$, serum with variant isoenzyme pattern. This variant pattern was observed in 6 of 54 normal $(N)$ subjects, 4 of 20 obligate $\mathrm{CF}$ heterozygotes $(\mathrm{H})$, and 2 of $8 \mathrm{CF}$ patients with adequate $P$ function. Analysis of the variant pattern frequency in the $C F$, $\mathrm{H}$, and $\mathrm{N}$ populations by the $\chi^{2}$ test confirms the conclusion of Schiotz and Magid (17) that the variant pattern is not significantly associated with the CF genotype.
Table 1. Pancreatic function and serum amylase $P$ isoenzyme

\begin{tabular}{cccc} 
& \multicolumn{3}{c}{ levels in $C F$ patients } \\
Group & $\begin{array}{c}\text { Age } \\
\text { (years) }\end{array}$ & $n$ & $\begin{array}{c}\text { Serum } P \text { isoenzyme } \\
\text { (units/liter) }\end{array}$ \\
${ }^{1}$ & $6-27$ & 6 & $1.6 \pm 0.7^{2}$ \\
& & & $(0.6-2.7)^{3}$ \\
CF-B ${ }^{4}$ & $5-27$ & 6 & $12.6 \pm 6.0^{5}$ \\
& & & $(4.6-21.0)$
\end{tabular}

' $\mathrm{CF}$ patients with pancreatic insufficiency confirmed by pancreatic secretory studies with duodenal intubation (3).

${ }^{2}$ Mean \pm S.D.

${ }^{3}$ Numbers in parentheses, range.

${ }^{4} \mathrm{CF}$ patients with adequate pancreatic function demonstrated by absence of steatorrhea. These patients do not use any enzyme replacement therapy.

${ }^{5} P<0.001$, Wilcoxon Rank Sum test.

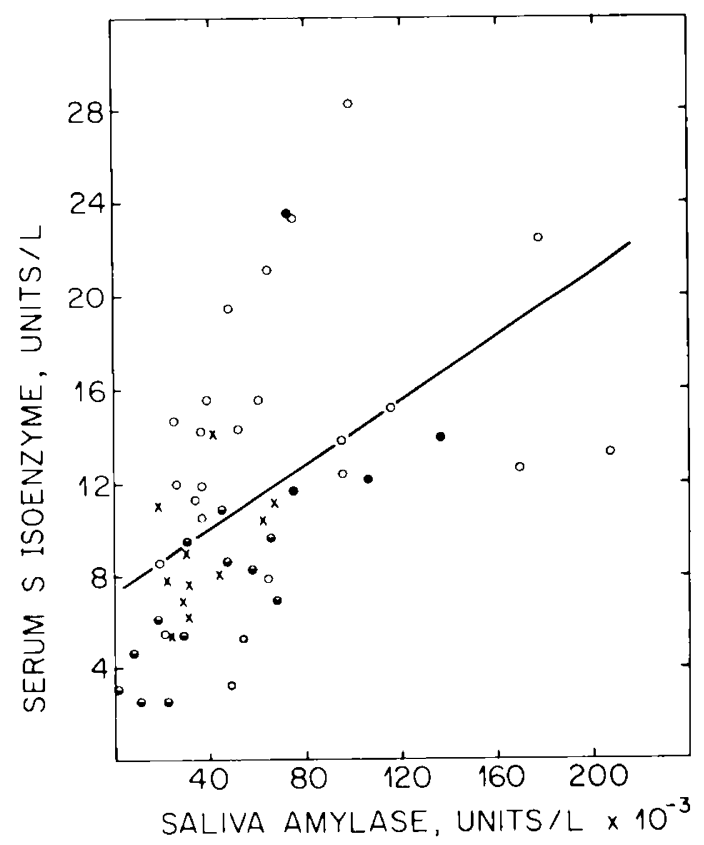

Fig. 2. Correlation of serum $S$ isoenzyme and saliva amylase activities. Points correspond to 51 pairs of serum and saliva samples. $\times, N$ samples; ๑. H samples, O, CF-A samples; $\bullet$, CF-B samples. Linear regression analysis gives $S$ (units/liter) $=7.2+6.8 \times 10^{\text {s. }}$ saliva amylase (units/liter). $r=0.53 ; P<0.001$. Linear regression was found to fit the data about as well as equations of the form $\mathrm{y}=\mathrm{a}+\mathrm{b} \log \mathrm{x}(r=0.57)$ and $\mathrm{y}=\mathrm{a}+\mathrm{bx}$ $(r=0.56)$.

that serum $\mathrm{S}$ isoenzyme levels can be used as a measure of salivary gland amylase secretion in $\mathrm{CF}, \mathrm{H}$, and $\mathrm{N}$ samples.

An alternate explanation for increased $S$ isoenzyme levels in $C F$ patient sera would be increased output of amylase by the diseased lung. This possibility is suggested by recent reports of increased $\mathrm{S}$ isoenzyme levels in cases of pneumonia and cancer ( $c f .21)$. Therefore, we analyzed $S$ isoenzyme levels as a function of the $X$ ray and the pulmonary components of the patient's Schwachman score. There was no correlation between either of these indices of lung disease and the $\mathrm{S}$ isoenzyme levels: $r=-0.25$ and $-0.16 ; P$ $>0.05$, for both. Thus, lung amylase output secondary to lung disease is not a primary contributor to serum $S$ isoenzyme levels in CF. Instead, based on the significant correlation between serum $S$ and saliva amylase levels, we conclude that saliva amylase output is the primary source of serum $\mathrm{S}$ isoenzyme in CF patients.

To determine the relationship between pancreatic and salivary amylase output in $C F$, we analyzed total serum amylase $S$ and $P$ isoenzyme activity with respect to $\mathrm{CF}$ genotype and pancreatic function. Inasmuch as there is still uncertainty about the normal 
range of serum amylase isoenzyme distribution below 5 years of age $(15,19,23)$, only samples from donors aged 5 years and over were analyzed. Mean values for total serum amylase, $\% P$, and $P$ and $S$ isoenzyme activity for the $\mathrm{CF}, \mathrm{H}$, and $\mathrm{N}$ groups are listed in Table 2.

The CF patients with pancreatic insufficiency have normal total serum amylase. In these subjects, $P$ isoenzyme activity is greatly reduced, consistent with the diagnosis of pancreatic acinar failure. In contrast, $S$ isoenzyme activity is significantly elevated and accounts for the normal level of total serum amylase. The amylase content of saliva is also elevated in this group.

The CF patients with adequate pancreatic function have elevated total serum amylase activity. The increased serum amylase is the result of significant increases in both the $P$ and $S$ isoenzyme activities. In parallel with increased serum $S$ activity, saliva amylase activity is also elevated in this group.

The $\mathrm{H}$ group (parents of CF patients) has total serum amylase activity within the normal range, but an altered distribution of $P$ and $S$ isoenzyme, with increased $P$ isoenzyme activity and increased mean \%P. However, there is overlap between the $\mathrm{H}$ and $N$ range of absolute and relative $P$ values (vide infra).

The relationship between $S$ and $P$ isoenzyme levels in CF genotypes was further analyzed with a scatter plot (Fig. 3). S and $P$ levels in $N$ sera are significantly correlated, with $P<0.001$ (Fig. $3 A$ ). $\mathrm{S}$ and $\mathrm{P}$ levels are also correlated in $\mathrm{H}$ sera with $P<0.01$ (Fig. $3 B$ ). Analysis of variance for $P$ versus $S$ indicates that the $H$ population is significantly different from the $\mathrm{N}$ population: $F=$ 4.47; $P<0.02$. Compared to $N$ samples, the $H$ samples have greater $P$ activity for a given $S$ activity. This shift accounts for the observed increase in \% $\mathrm{P}$ values in $\mathrm{H}$ samples.

The relationship between $S$ and $P$ values differs in the two $C F$ groups. In sera of CF patients with adequate pancreatic function, the increases in $\mathrm{S}$ and $\mathrm{P}$ values are correlated (Fig. $3 C$, solid circles). Sera of CF patients with pancreatic insufficiency have a similar range of $S$ values, but all have very low $P$ values, so there is no correlation between $\mathrm{S}$ and $\mathrm{P}$ values in this group (Fig. $3 C$, open circles).

\section{DISCUSSION}

These studies demonstrate that serum $P$ and $S$ amylase isoenzyme levels in CF patients correlate with their pancreatic and salivary gland amylase output and therefore that $P$ and $S$ levels can be used as measures of pancreatic and salivary exocrine function in the $C F$ patient. Our finding that total serum amylase, the sum of $P$ and $S$ activity, is normal in CF patients with pancreatic insufficiency and elevated in patients with adequate pancreatic function (Table 2) confirms the recent report of Davidson et al. (5). These authors found normal total serum amylase in CF patients with steatorrhea, but below normal total serum amylase in Schwachman's syndrome patients. Inasmuch as the total serum amylase in both of these patient groups with pancreatic insufficiency is comprised virtually completely of $S$ isoenzyme, Davidson $e t$ al. concluded that elevated $S$ isoenzyme is intrinsic to $\mathrm{CF}$ and not a secondary manifestation of pancreatic insufficiency. Our direct measurements of $\mathbf{S}$ isoenzyme and salivary amylase activity in $C F$ patients confirm that these are elevated in $C F$ patients regardless of pancreatic function. Thus, salivary amylase hypersecretion is not secondary to decreased pancreatic amylase production.

Our finding that $P$ isoenzyme levels reflect pancreatic amylase output confirms the earlier reports of Taussig et al. (22) and Wolf et al. (24) that serum $P$ isoenzyme levels are useful for the diagnosis of pancreatic insufficiency in CF. However, serum amylase isoenzyme levels are low in normal newborns $(15,19,23)$. Adult levels have been reported to be reached as early as 8 months (23) and as late as 10 to 15 years of age (19). This disagreement on the normal development of serum amylase isoenzyme activity needs to be resolved, and the normal ranges of $P$ and $S$ isoenzymes need to be defined for the 0-to 5-year group before application of serum amylase isoenzyme levels for the diagnosis of pancreatic insufficiency in the younger child with $\mathrm{CF}$.

Our data further show that pancreatic as well as salivary amylase seems elevated in CF patients with adequate pancreatic function, i.e., those with residual acinar function. This is consistent with reports of recurrent acute pancreatitis in CF patients who do not have pancreatic insufficiency (18). It suggests that increased concentration of macromolecules in the pancreatic duct may precede the plugging of these ducts in those patients who ultimately develop pancreatic insufficiency. However the fact that pancreatic hypersecretion is present in CF patients with adequate pancreatic function is evidence that hypersecretion per se, is not a sufficient explanation for duct plugging and the development of pancreatic insufficiency and that some other contributing events must also be involved.

There is some indication that serum $P$ isoenzyme levels may be elevated in the $C F$ neonate. Studies with autopsy material have shown that pancreatic acinar changes can begin in utero for the $\mathrm{CF}$ fetus (10), although the progression of pancreatic disease after birth can be very rapid or be clinically indiscernible (11). Crossley $e t$ al. (4) have reported a study of five neonates with CF whose initial serum levels of immunoreactive trypsin were markedly

Table 2. Serum amylase isoenzymes and saliva amylase in $C F$ genotypes ${ }^{1}$

\begin{tabular}{|c|c|c|c|c|c|c|c|c|}
\hline \multirow[b]{2}{*}{ Group } & \multirow[b]{2}{*}{$\mathrm{Age}^{2}$} & \multicolumn{5}{|c|}{ Serum } & \multicolumn{2}{|c|}{ Saliva } \\
\hline & & $n$ & $\begin{array}{c}\begin{array}{c}\text { Total } \\
\text { (units/liter) }\end{array} \\
\end{array}$ & $\% \mathbf{P}^{3}$ & $\begin{array}{c}\mathrm{P} \\
\text { (units/liter) }\end{array}$ & $\begin{array}{c}\mathbf{S} \\
\text { (units/liter) }\end{array}$ & $n$ & $\begin{array}{c}\text { Total } \\
\text { (units/ml) }\end{array}$ \\
\hline$N$ & $5-47$ & 50 & $13.8 \pm 4.2^{4}$ & $42 \pm 7$ & $5.8 \pm 1.7$ & $8.1 \pm 2.7$ & 43 & $32 \pm 13$ \\
\hline$C F \cdot A^{5}$ & $5-35$ & 28 & $13.7 \pm 5.4$ & $10 \pm 6^{6}$ & $1.3 \pm 0.6^{65}$ & $12.5 \pm 5.3^{6 i}$ & 22 & $68 \pm 49^{i}$ \\
\hline$C F \cdot B^{i}$ & $5-27$ & 8 & $24.5 \pm 9.8^{\star}$ & $49 \pm 11$ & $12.0 \pm 5.3^{\mathrm{K}}$ & $12.5 \pm 5.2^{4}$ & 4 & $98 \pm 30^{6}$ \\
\hline $\mathrm{H}$ & $26-47$ & 19 & $14.3 \pm 4.4$ & $51 \pm 9^{6 i}$ & $7.2 \pm 2.1^{111}$ & $7.1 \pm 2.8$ & 34 & $40 \pm 27$ \\
\hline
\end{tabular}

'Groups were compared using the Wilcoxon Rank Sum test. Male and female values were not significantly different.

"Comparison of values for 5 to 14,15 to 24,25 to 35 , and 36 to 47 year olds indicated no difference in control values for these different age groups; therefore, all values were combined for comparison to CF and $\mathrm{H}$ values. For $\mathrm{CF}$ samples, serum amylase values were not significantly different for the different age groups, but saliva amylase was most elevated in the 15- to 24 -year group as reported previously (8). The mean age of the patient population was $18 \pm 8$ years; that of the control population of the same age ( 5 to 35 years) was also $18 \pm 8$ years $(n=45)$.

"Mean value calculated from individual $\% P$ values, not from the mean $P$ and mean total amylase values.
${ }^{4}$ Mean \pm S.D.

${ }^{5} \mathrm{CF}$ patients with pancreatic insufficiency.

" $P<0.001$ versus $\mathrm{N}$.

${ }^{i} \mathrm{CF}$ patients with adequate pancreatic function.

${ }^{*} P<0.002$ versus $N$.

${ }^{9} P<0.01$ versus $\mathrm{N}$.

${ }^{10} P<0.05$ versus $N$ 


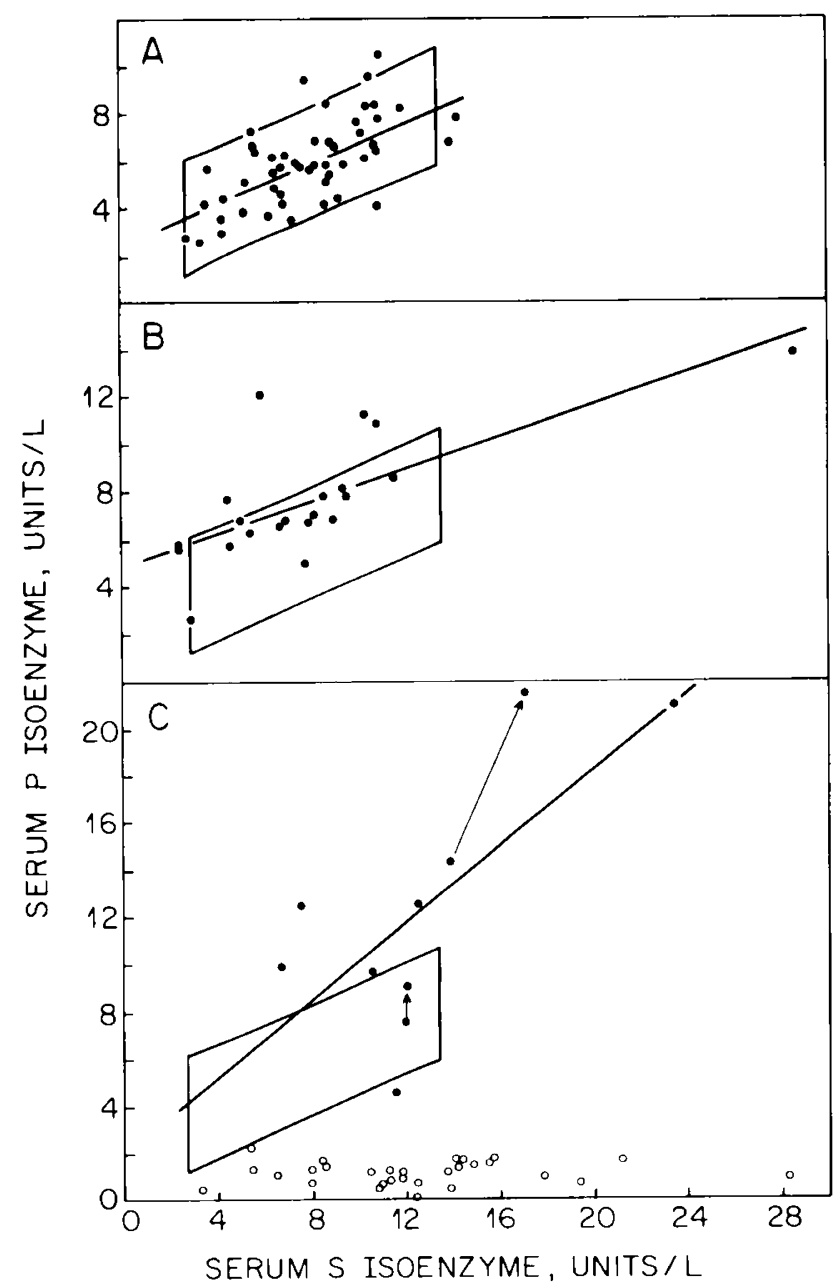

Fig. 3. Correlation of serum $P$ and $S$ isoenzyme levels in $C F$ genotypes. $A$, normal sera. The line is the linear least squares fit: $\mathrm{P}=2.43+0.43 \mathrm{~S}$; $n=50 ; r=0.64 ; P<0.001$. The box encloses the $95 \%$ confidence limits for individual $P$ values for the $P$ versus $S$ regression line, bounded by the $95 \%$ confidence limits for normal $\mathrm{S}$ isoenzymes levels; $B$, heterozygote sera. The line is the linear least squares fit to the $\mathrm{H}$ data: $\mathrm{P}=4.76+0.34$ $\mathrm{S} ; n=21 ; r=0.57 ; P<0.01$. The box is the same as in $A ; C, C F$ sera. $\bullet$, patients with adequate pancreatic function: $O$, patients with pancreatic insufficiency. Arrows connect points for sequential serum samples from the same individual. Line is the linear least squares fit to the solid points: $P$ $=1.72+0.83 \mathrm{~S} ; n=10, r=0.72, P<0.02$.

elevated. These levels decreased gradually during the first months of life in parallel with development of pancreatic insufficiency. This suggests that initial acinar dysfuntion in CF results in elevated ductal pancreatic enzyme concentration and is followed in most cases by development of pancreatic acinar insufficiency. We suggest that serum levels of pancreatic enzymes, such as immunoreactive trypsin and $P$ amylase isoenzyme, may be useful for testing this model for pancreatic disease progression in CF.

The altered exocrine gland regulation demonstrated in $\mathrm{CF}$ patients seems to be partially expressed in the CF heterozygote. Thus, sera of parents of $C F$ patients have increased values of $\% P$ isoenzyme and absolute pancreatic amylase (Table 2). Our observations confirm those of Skude and Kollberg (20) and Schiotz and Magid (17), indicating that an increased mean $\% \mathrm{P}$ is a consistent finding in the $\mathrm{H}$ population. However, the $\mathrm{H}$ and $\mathrm{N}$ ranges overlap so that serum amylase isoenzyme levels cannot be used to identify CF heterozygotes.

The quantitative relationships between serum $S$ and $P$ isoenzyme levels in $\mathrm{CF}, \mathrm{H}$, and $\mathrm{N}$ samples (Fig. 3) suggest several deductions regarding the regulation of amylase secretion. First, we conclude that there is no feedback mechanism for interaction between the salivary and pancreatic glands to control total serum amylase levels because the greatly reduced levels of $P$ isoenzyme in sera of CF patients with pancreatic insufficiency do not induce a uniform increase in salivary amylase output. This conclusion is supported by the low total serum amylase levels in patients with Schwachman's syndrome (6). Second, the normal pancreas and salivary gland seem to respond in a fixed proportion to common regulatory agents because there is a good correlation between $\mathrm{S}$ and $\mathrm{P}$ levels in $\mathrm{N}$ sera (Fig. $3 A$ ). In $\mathrm{H}$ sera, $\mathrm{P}$ and $\mathrm{S}$ are also correlated, but $P$ values are higher than in $N$ sera of the same $S$ value (Figure $3 B$ ). We speculate that this may be due to an extra agent in the $\mathrm{H}$ group present at concentrations which stimulate pancreatic but not salivary amylase secretion.

In sera of $C F$ patients with pancreatic function, both $S$ and $P$ isoenzyme levels are elevated compared to $\mathrm{N}$ levels (Figure $3 C$ ). The range of $P$ and $S$ levels indicates that the $C F$ glands are able to respond to secretory stimuli, but the resultant secretions contain increased amylase activity. Thus, the correlation between $S$ and $P$ activity further supports a generalized defect in regulation of exocrine secretion in CF. We suggest that the altered regulation could be due to an extra, stimulatory agent which acts on both the pancreas and the salivary glands. We further speculate that this agent could be one of the "CF factors" ( $c f$. Ref. 6), and that the pancreas responds to lower concentrations of this factor than does the salivary gland. This would account for both the greater involvement of the pancreas in the CF disease process and for the altered pancreatic but not salivary amylase secretion in the $C F$ heterozygote. A test of this hypothesis requires isolation of the $\mathrm{CF}$ factor(s) and testing of their effect on exocrine gland secretory regulation.

\section{REFERENCES AND NOTES}

1. Berk. J. E. and Fridhandler, L.: Clinical application of amylase isoenzyme analysis. Am. J. Gastroenterol., 63: 457 (1975)

2. Chernick, W. S., Eichel, H. J., and Barbero, G. J.: Submaxillary salivary enzymes as a measure of glandular activity in cystic fibrosis. J. Pediatr., 05: 694 (1964).

3. Cox, K. L.. Isenberg. J. N.. Osher, A. B.. and Dooley, R. R.: The effect of cymetidine on maldigestion in cystic fibrosis. J. Pediatr., 94: 488 (1979).

4. Crossley, J. R.. Elliott. R. B., and Smith, P. A.: Dried-blood spot screening for cystic fibrosis in the newborn. Lancet, 1: 472 (1979).

5. Davidson, G. P., Koheil. A., and Forstner, G. G.: Salivary amylase in cystic fibrosis: a marker of disordered autonomic function. Pediatr. Res. 12: 967 (1978).

6. DiSant Agnese, P. A., and Davis, P. B.: Research in cystic fibrosis. N. Engl. J. Med.. 295: 481, 534, and 597 (1976).

7. Gillard, B. K.: Quantitative gel-electrophoretic determination of serum isoenzyme distributions. Clin. Chem.. 25: 1919 (1979).

8. Gillard, B. K. Markman, H. C. and Feig. S. A.: Differences between cystic fibrosis and normal saliva a-amylase as a function of age and sex. Pediatr. Res., 12: 868 (1978).

9. Gillard, B. K., Simbala, J., and Pedersen, S.: Amylase isoenzymes in serum and saliva of cystic fibrosis patients: Evidence for increased exocrine secretion. Cystic Fibrosis Club Abstracts, 20: 9 (1979)

10. Imrie. J. R.. Fagen. D. G.. and Sturgess. J. M.: Quantitative evaluation of the development of the exocrine pancreas in cystic fibrosis and control infants. Am. J. Pathol. 95: 697 (1979).

11. Kopito, L. E.. Schwachman. H., Vawter. C. F., and Edlow. J.: The pancreas in cystic fibrosis: chemical composition and comparative morphology. Pediatr. Res.. 10: 742 (1976).

12. Merritt. A. D.. Rivas, M. L.. Bixler, D.. and Newall, R.: Salivary and pancreatic amylase. Electrophoretic characterizations and genetic studies. Am. J. Hum. Genet.. 25: 510: (1973).

13. O'Donnell, M., Fitzgerald. O.. and McGieeney, K. F.: Differential serum amylase determination by use of an inhibitor, and design of a routine procedure. Clin. Chem.. 23: 560 (1977).

14. Otsuki, M.. Saeki. S., Yuu, H.. Maeda. M., and Baba. S.: Electrophoretic pattern of amylase isoenzymes in serum and urine of normal persons. Clin. Chem., 22: 439 (1976).

15. Otsuki, M., Yuu, H., Saeki, S., and Baba. S.: The characteristics of amylase activity and the isoamylase pattern in serum and urine of infants and children. Eur. J. Pediatr., 125: 175 (1977).

16. Salt, W. B., and Schenker, S.: Amylase-its clinical significance: a review of the literature. Medicine (Baltimore). 55: 269 (1976).

17. Schiotz, P. O., and Magid, E.: Serum pancreatic isoamylases in the diagnosis of cystic fibrosis heterozygotes: a non-valuable test. Clin. Genet.. //: 43 (1977).

18. Schwachman, H., Leventhal, E.. and Khaw, K. T.: Recurrent acute pancreatitis in patients with cystic fibrosis with normal pancreatic enzymes. Pediatrics, 55: $86(1975)$.

19. Skude. G.: Sources of serum isoamylases and their normal range of variation 
with age. Scand. J. Gastroenterol.. 10: 477 (1975)

20. Skude. G., and Kollberg, H.: Serum isoamylases in cystic fibrosis. Acta Paediatr. Scand., 65: 145 (1976).

21. Sudo, K., and Kanno, T.: Properties of the amylase produced in carcinoma of the lung. Clin. Chim. Acta.. 73: 1 (1976).

22. Taussig. L. M., Wolf, R. O., Woods, R. E.. and Deckelbaum, R. J.: Use of serum amylase isoenzymes in evaluation of pancreatic function. Pediatrics, 54: 229 (1974).

23. Tye. J. G., Karn, R. C., and Merritt, A. D.: Differential expression of salivary (Amy $)$ and pancreatic (Amy.2) human amylase loci in prenatal and postnatal development. J. Med. Genet., I3: 96 (1976).

24. Wolf, R. O., Taussig, L. M., Ross, M. E., and Wood, R. E.: Quantitative evaluation of serum pancreatic isoamylases in cystic fibrosis. J. Lab. Clin.
Med.. 87: 164 (1976).

25. Presented in part at the (ystic Fibrosis Club Meeting. Atlanta, May I. 1979 (9). 26. The authors thank Drs. Alan Osher and Paul Pollack for the clinical evaluation of pancreatic function in the CF patients. Drs. Kenneth Cox and Nevin Isenberg for the results of their pancreatic secretory studies on some of the patients (3). Sue Pedersen and Grace Sabig for sample collection, and Deborah Kim for manuscript preparation.

27. Requests for reprints should be addressed to: B. K. Gillard. Ph.D., Department of Pediatrics, UCLA School of Medicine, Los Angeles. CA 90024 (USA).

28. This research was supported by a Research Grant from the Cystic Fibrosis Foundation and NIH Grant AM 25107.

29. Received for publication December 3. 1979

30. Accepted for publication February 15, 1980

Copyright (1) 1980 International Pediatric Research Foundation. Inc. $0031-3998 / 80 / 1411-1168 \$ 02.00 / 0$ 